OPEN ACCESS

Edited by:

Vinay V. Parikh,

Temple University, United States

Reviewed by:

Ezgi Hacisuleyman,

The Rockefeller University,

United States

Ganna Palagina,

Harvard Medical School,

United States

*Correspondence:

Xiaodong Liu

xiaodongsheldon@outlook.com

Linqing Miao

linqingmiao@gmail.com

${ }^{\dagger}$ Co-first authors

Received: 08 May 2019

Accepted: 24 September 2019

Published: 16 October 2019

Citation:

Chen J, Zhang L, Liu L, Yang $X$, Wu F, Gan X, Zhang R, He Y, LV Q, Fu H, Zhou L, Zhang J, Liu A, Liu X and Miao L (2019) Acupuncture

Treatment Reverses Retinal Gene Expression Induced by Optic Nerve Injury via RNA Sequencing Analysis.

Front. Integr. Neurosci. 13:59.

doi: 10.3389/fnint.2019.00059

\section{Acupuncture Treatment Reverses Retinal Gene Expression Induced by Optic Nerve Injury via RNA Sequencing Analysis}

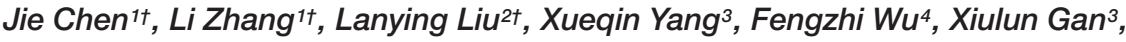 \\ Rong Zhang ${ }^{1}$, Yinjia He ${ }^{1}$, Qiuyi Lv' ${ }^{1}$, Haonan $\mathrm{Fu}^{1}$, Ling Zhou ${ }^{1}$, Jiaxi Zhang ${ }^{1}$, Anming Liu', \\ Xiaodong Liu ${ }^{1 *}$ and Linqing Miao ${ }^{5 *}$
}

\begin{abstract}
${ }^{1}$ School of Acupuncture-Moxibustion and Tuina, Beijing University of Chinese Medicine, Beijing, China, ${ }^{2}$ The Third Affiliated Hospital, Beijing University of Chinese Medicine, Beijing, China, ${ }^{3}$ School of Traditional Chinese Medicine, Beijing University of Chinese Medicine, Beijing, China, ${ }^{4}$ Journal Center, Beijing University of Chinese Medicine, Beijing, China, ${ }^{5}$ Beijing Advanced Innovation Center for Intelligent Robots and Systems, Beijing Institute of Technology, Beijing, China
\end{abstract}

Glaucoma and traumatic optic nerve crush (ONC) injury result in progressive loss of retinal ganglion cells (RGCs) and defects in visual function. In clinical trials of Traditional Chinese Medicine, acupuncture has been widely used for the treatment of ocular diseases. However, the molecular mechanisms of acupuncture treatment are still unclear. In this study, we used technique of RNA sequencing (RNA-seq) to study the effects of acupuncture treatment on retinal transcriptome after axotomy injury. RNA-seq results revealed that 436 genes including 31 transcription factors (TFs) were changed after injury, among them were many well-known neural degeneration related TFs such as Jun, Ddit3, Atf3, and Atf4. Interestingly, acupuncture treatment at acupoint GB20 (Fengchi) significantly reversed a series of differential expressed genes (DEGs) induced by optic nerve injury. While treatments at BL1 (Jingming) or GB20 sham control acupoint-GV16 (Fengfu), led to limited DEG reversal. In contrast, treatments at these two sites further enhanced the trend of DEG expression induced by axotomy injury. At last, retina immunostaining results revealed that only GB20 acupoint treatment increased RGC survival, in consistent with RNA-seq results. Therefore, our study first reported that acupuncture treatment regulated retinal transcriptome and reversed the gene expression induced by axotomy injury, and GB20 acupoint treatment increased RGC survival, which will provide novel therapeutic targets for treatment of ocular diseases.

Keywords: optic nerve crush, retinal ganglion cells, acupuncture, RNA sequencing, transcriptome, GB20 (Fengchi), BL1 (Jingming), GV16 (Fengfu)

\section{INTRODUCTION}

Chronic eye diseases such as glaucoma and optic neuritis result in progressive loss of retinal ganglion cells (RGC) and blindness finally (Shindler et al., 2008; Almasieh et al., 2012). Traumatic injury such as optic nerve crush (ONC) also cause serious RGC death (Sanchez-Migallon et al., 2016). Previous studies show that both chronic and traumatic optic nerve injury induce endoplasmic reticulum (ER) stress in RGC soma, while manipulation of ER stress pathway promoted the survival of RGC (Yang et al., 2016; Huang et al., 2017). The induction of upregulation of pro-apoptotic TFs such as JUN and DDIT3 after injury leads to progressive RGC death 
(Huang et al., 2017; Syc-Mazurek et al., 2017a,b; Welsbie et al., 2017). Acupuncture has been widely used to treat many kinds of pains such as neck and shoulder pain, lower back pain worldwide (MacPherson et al., 2017; Koh et al., 2018). Acupuncture has also been used for treatment of ocular diseases, including glaucoma, age-related macular degeneration (AMD), retinitis pigmentosa, etc (Jiao, 2011; Xu et al., 2012; Law and Li, 2013; Xu and Peng, 2015). In Traditional Chinese Medicine, GB20 and BL1 are the common acupoints selected for acupuncture treatment of ocular diseases (Qin et al., 2015; Xu and Peng, 2015). Although acupuncture is widely used for ocular therapy, the effect of acupuncture treatment on regulation of retinal transcriptome especially those changed by ONC injury is totally unknown. RNA sequencing (RNA-seq) is a new technique effective in identifying numerous genes regulated by specific treatment. In this study, we will use ONC crush injury mouse model and RNA-seq technique to analyze retinal samples with and without optic nerve injury and screen for the upregulated and downregulated TFs 2 days after ONC injury, and also identified those injury-induced genes reversed after acupuncture treatment at two different acupoints.

\section{RESULTS}

\section{Expression Analysis}

To investigate expression differentiation, we extract RNA from intact retina (WT), axotomized retina (ONC), and axotomized retina with acupuncture treatment at acupoint GB20 (ONC-F) or BL1 (ONC-J), 2 days after ONC. The actual and schematic acupuncture sites were shown in Figures 1A,B. RNA integrity of each sample was assessed by Bioanalyzer 2100 system. Sample total reads ranged from 40.8 to 69.5 million, and mapping rate ranged from 95.4 to $96.6 \%$.

We then used feature Counts v1.5.0-p3 to calculate expected number of Fragments Per Kilobase of transcript sequence per Millions base pairs sequenced (FPKM) of each sample, which represent relative gene expression abundance. The boxplot result showed that the overall distribution of the FPKM values were consistent among samples, suggesting that the RNA-seq data were reproducible (Figure 2A). Principal component analysis (PCA) showed that wild-type (WT) control formed clear cluster apart from injury groups, and ONC with or without acupuncture treatment formed one big cluster, ONC with sham treatment was separated from acupuncture treatment groups (Figure 2B). Differentially expressed genes (DEG) cluster analysis showed that acupuncture treatment groups were clustered together and separated from ONC control, and all the ONC groups with or without acupuncture treatments were separated from WT control group (Figure $2 \mathrm{C}$ ).

\section{Differentially Expressed Genes After ONC}

To identify candidate genes regulated by axon injury 2 days after axotomy, we performed differential expression analysis using the DESeq2 R package (1.16.1). Genes with $\mid \log _{2}$ FoldChange $\mid>0$ and adjusted $p$-value $<0.05$ were assigned as differentially expressed. We then identified total 436 differentially expressed genes (DEG), with 191 genes upregulated and 245 genes downregulated (Figure 3A). Enriched gene ontology analysis results showed that the most upregulated gene categories were cell adhesions and junctions (Figure 3B); while the most downregulated gene categories were: axon, postsynapse and transmembrane transports (Figure 3C). The 20 most significantly upregulated and downregulated DEGs after ONC are shown in Table $\mathbf{1 .}$

\section{Transcription Factors Up-Regulated After ONC}

Since TFs play important roles in both axon development and injury-induced degeneration, to narrow down the candidate DEGs, we next identified TFs upregulated and downregulated after axon injury, which result in 15 upregulated TFs and 16 downregulated TFs (Table 2). The upregulated TFs include many pro-apoptotic molecules involved in ER stress response, such as Atf4 and Ddit3 (Woo et al., 2012; Hiramatsu et al., 2014; Joo et al., 2015; Walter et al., 2018); Creb5 and Kdr in PI3K-Akt signaling pathway (Bellon et al., 2010; Kay et al., 2013; Wu et al., 2017); Jun and Cebpb in TNF signaling pathway (Mayer et al., 2013; Cao et al., 2018); Ddit3, Atf4 and Jun in apoptosis pathway (Holland et al., 2016; Ishizawa et al., 2016; Syc-Mazurek et al., 2017b), and most of these upregulated TFs resulted in RGC death.

\section{Reversal of Injury Induced DEGs by Acupuncture Treatments}

To study the effect of acupuncture treatment after ONC injury, we performed acupuncture treatments 3 times after ONC at acupoint GB20 (Fengchi), BL1 (Jingming) or sham point GV16 (Fengfu) (Figure 1B), respectively. RNA-seq analysis showed that among the injury induced up-regulated DEGs, 20 DEGs were reversed by GB20 treatment with only 6 DEGs further up-regulated (Figure 4A), the reversal DEG number was 11 in BL1 and 14 in GV16 treatments, however, both BL1 and GV16 treatments further up-regulated even more DEGs, which was 24 in BL1 and 52 in GV16 (Figures 4B,C). Among the injury induced down-regulated DEGs, 21 DEGs were reversed by GB20 treatment without further down-regulated DEGs (Figure 4D), the reversal DEG number was 19 in BL1 with only 2 DEGs further down-regulated (Figure 4E), however, in GV16 treatments, the reversal number is 32 vs. 42 further down-regulated DEGs (Figure 4F). The average FPKM values of the 436 DEGs of all sample groups and the reversal percentages of all the DEGs after BL1, GB20, and GV16 treatments were listed in Supplementary Table S1.

\section{Validation DEGs With qRT-PCR}

To verify the reliability of RNA-seq result, we selected four important TFs reported to be upregulated after axon injury (Jun, Ddit3, Atf3, and Sox11) (Hu et al., 2012; Fagoe et al., 2015; Holland et al., 2016; Huang et al., 2017; Lu et al., 2017; Norsworthy et al., 2017; Struebing et al., 2017; Li et al., 2018; Wong et al., 2018) to perform qRT-PCR test. Results showed that all the four TFs were significantly upregulated after ONC injury (Figures 5A-D), consistent with previous reports. We then 

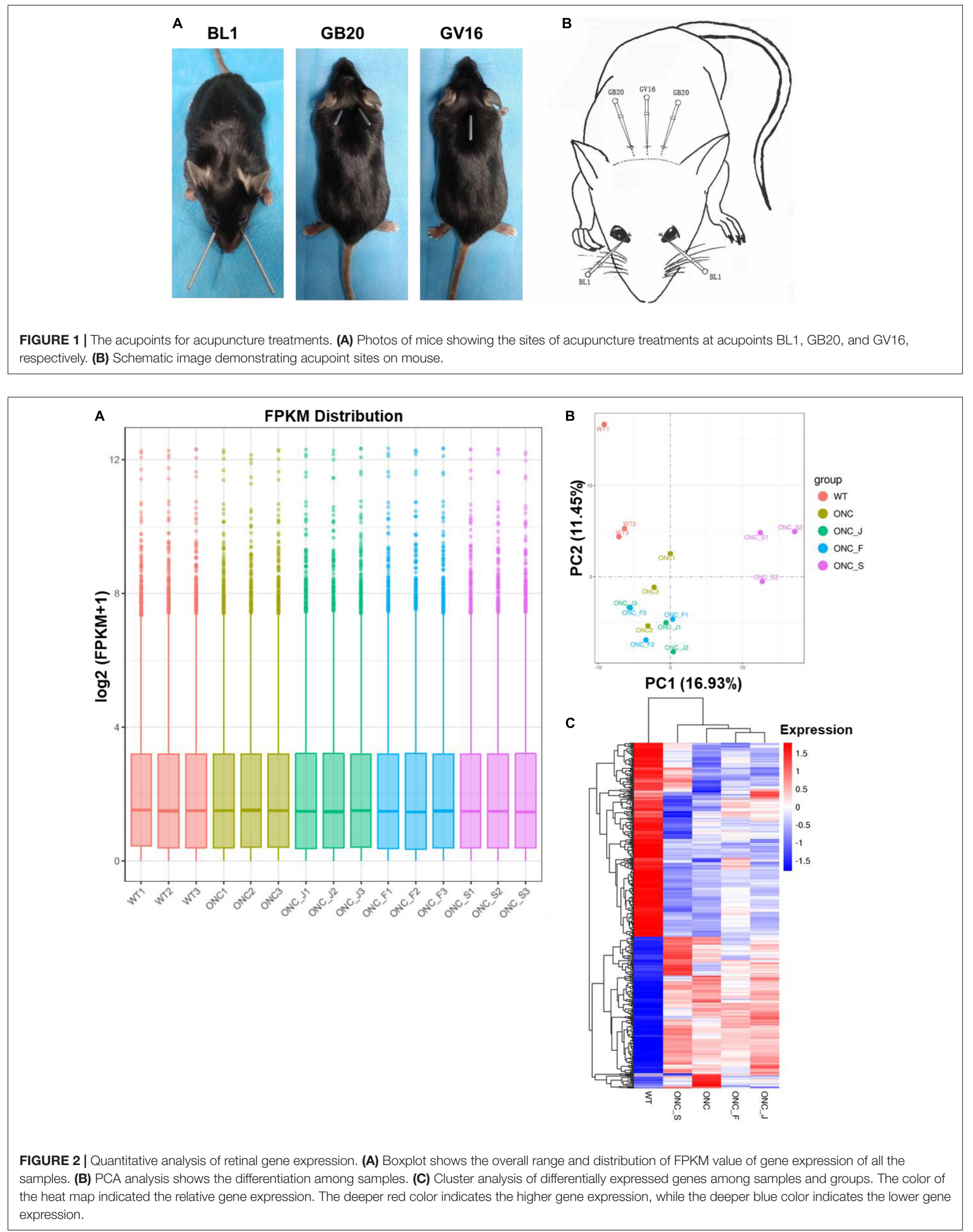
TABLE 1 | Top 20 upregulated and 20 downregulated genes induced by ONC injury.

\begin{tabular}{|c|c|c|c|c|}
\hline Gene name & $\begin{array}{l}\quad \log _{2} \\
\text { FoldChange }\end{array}$ & Padj & Gene id & Gene description \\
\hline \multicolumn{5}{|l|}{ Up-regulated } \\
\hline Sprr1a & 10.2 & 3.6E-15 & ENSMUSG00000050359 & Small proline-rich protein 1A [Source:MGI Symbol;Acc:MGl:106660] \\
\hline Gm29374 & 7.3 & 5.3E-07 & ENSMUSG00000099839 & Predicted gene 29374 [Source:MGl Symbol;Acc:MGl:5580080] \\
\hline Gm38403 & 6.8 & 4.1E-05 & ENSMUSG00000112854 & Predicted gene, 38403 [Source:MGI Symbol;Acc:MGL:5621288] \\
\hline Ecel1 & 6.7 & $3.8 \mathrm{E}-84$ & ENSMUSG00000026247 & Endothelin converting enzyme-like 1 [Source:MGI Symbol;Acc:MGl:1343461] \\
\hline Mmp12 & 6.2 & $1.3 E-49$ & ENSMUSG00000049723 & Matrix metallopeptidase 12 [Source:MGI Symbol;Acc:MGI:97005] \\
\hline Cox6a2 & 4.9 & 4.0E-05 & ENSMUSG00000030785 & Cytochromec oxidase subunit 6A2 [Source:MGI Symbol;Acc:MGl:104649] \\
\hline Cyb5r2 & 3.8 & 1.4E-05 & ENSMUSG00000048065 & Cytochromeb5 reductase 2 [Source:MGl Symbol;Acc:MGl:2444415] \\
\hline Tmc1 & 3.8 & $1.8 \mathrm{E}-05$ & ENSMUSG00000024749 & $\begin{array}{l}\text { Transmembrane channel-like gene family } 1 \text { [Source:MGl } \\
\text { Symbol;Acc:MGl:2151016] }\end{array}$ \\
\hline Chac1 & 3.3 & $1.9 \mathrm{E}-51$ & ENSMUSG00000027313 & ChaC, cation transport regulator 1 [Source:MGI Symbol;Acc:MGl:1916315] \\
\hline Gm47593 & 3.1 & $2.5 \mathrm{E}-04$ & ENSMUSG00000112058 & Predicted gene, 47593 [Source:MGI Symbol;Acc:MGl:6096640] \\
\hline AC167169.1 & 3.0 & 1.6E-06 & ENSMUSG00000117324 & Novel transcript, antisense to Dscam \\
\hline Plaur & 3.0 & 3.2E-02 & ENSMUSG00000046223 & Plasmnogen activator urokinase receptor [Source:MGI Symbol;Acc:MGI:97612] \\
\hline Top2a & 2.8 & 4.0E-02 & ENSMUSG00000020914 & Topoisomerase (DNA) II alpha [Source:MGI Symbol;Acc:MGl:98790] \\
\hline Hmox1 & 2.8 & 2.8E-02 & ENSMUSG00000005413 & Heme oxygenase 1 [Source:MGI Symbol;Acc:MGI:96163] \\
\hline Atf3 & 2.8 & $1.0 \mathrm{E}-67$ & ENSMUSG00000026628 & Activating transcription factor3 [Source:MGI Symbol;Acc:MGl:109384] \\
\hline Hrk & 2.7 & $8.2 \mathrm{E}-40$ & ENSMUSG00000046607 & $\begin{array}{l}\text { Harakiri, BCL2 interacting protein (contains only BH3 domain) [Source:MGI } \\
\text { Symbol;Acc:MGl:1201608] }\end{array}$ \\
\hline Sox11 & 2.7 & $2.0 \mathrm{E}-48$ & ENSMUSG00000063632 & SRY (sex determining region Y)-box 11 [Source MGI Symbol;Acc:MGI:98359] \\
\hline Cdkn1a & 2.6 & $1.3 \mathrm{E}-24$ & ENSMUSG00000023067 & $\begin{array}{l}\text { Cyclin-dependent kinase inhibitor 1A (P21) [Source:MGI } \\
\text { Symbol;Acc:MGl:104556] }\end{array}$ \\
\hline Lad1 & 2.4 & 3.6E-02 & ENSMUSG00000041782 & Ladinin [Source:MGI Symbol;Acc:MGl:109343] \\
\hline Tnfrsf12a & 2.4 & $2.7 \mathrm{E}-66$ & ENSMUSG00000023905 & $\begin{array}{l}\text { Tumor necrosis factor receptor superfamily, member 12a [Source:MGI } \\
\text { Symbol:Acc:MGl:1351484] }\end{array}$ \\
\hline \multicolumn{5}{|l|}{ Down-regulated } \\
\hline Scn4b & -3.38 & 4.1E-23 & ENSMUSG00000046480 & Sodium channel type IV beta [Source MGI Symbol;Acc:MGI:2687406] \\
\hline Ppp1r1c & -3.10 & $1.2 \mathrm{E}-03$ & ENSMUSG00000034683 & $\begin{array}{l}\text { Protein phosphatase 1, regulatory inhibitor subunit 1C [Source:MGl } \\
\text { Symbol;Acc:MGl:1923185] }\end{array}$ \\
\hline $\operatorname{lr} \times 4$ & -2.36 & $1.0 \mathrm{E}-14$ & ENSMUSG00000021604 & Iroguois homeobox 4 [Source:MGI Symbol;Acc:MGl:1355275] \\
\hline Gpr101 & -2.20 & 2.4E-02 & ENSMUSG00000036357 & G protein-coupled receptor 101 [Source MGI Symbol;Acc:MGI:2685211] \\
\hline Hapln1 & -2.16 & 5.7E-04 & ENSMUSG00000021613 & $\begin{array}{l}\text { Hyaluronan and proteoglycan link protein } 1 \text { [Source:MGl } \\
\text { Symbol;Acc:MGl:1337006] }\end{array}$ \\
\hline Efcab1 & -1.94 & $1.2 \mathrm{E}-02$ & ENSMUSG0C000068617 & EF-hand calcium binding domain 1 [Source:MGI Symbol;Acc:MGI:1914043] \\
\hline Pou4f2 & -1.88 & 1.6E-20 & ENSMUSG00000031688 & $\begin{array}{l}\text { POU domain, class 4, transcnption factor } 2 \text { [Source:MGl } \\
\text { Symbol;Acc:MGl:102524] }\end{array}$ \\
\hline Htr1b & -1.86 & $1.0 \mathrm{E}-14$ & ENSMUSG00000049511 & $\begin{array}{l}\text { 5-hydroxytryptamine (serotonin) receptor IB [Source:MGI } \\
\text { Symbol;Acc:MGl:96274] }\end{array}$ \\
\hline Ctxn3 & -1.82 & $6.2 E-11$ & ENSMUSG00000069372 & Cortexin 3 [Source:MGI Symbol;Acc:MGl:3642816] \\
\hline Kctd19 & -1.75 & 1.6E-03 & ENSMUSG00000051648 & $\begin{array}{l}\text { Potassium channel tetramerization domain containing } 19 \text { [Source:MGI } \\
\text { Symbol;Acc:MGl:3045294] }\end{array}$ \\
\hline D130079A08Rik & -1.72 & $2.5 \mathrm{E}-02$ & ENSMUSG00000115424 & RIKEN cDNA D130079A08 gene [Source:MGI Symbol;Acc:MGI:2444500] \\
\hline Tusc5 & -1.65 & 4.7E-38 & ENSMUSG00000046275 & Tumor suppressor candidate 5 [Source:MGI Symbol;Acc:MGl:3029307] \\
\hline Pvalb & -1.61 & $9.8 \mathrm{E}-12$ & ENSMUSG00000005716 & Parvalbumin [Source:MGI Symbol;Acc:MGl:97821] \\
\hline 2600014E21Rik & -1.52 & $4.2 \mathrm{E}-02$ & ENSMUSG00000100303 & RIKEN cDNA 2600014E21 gene [Source:MGI Symbol;Acc:MGI:1919384] \\
\hline Irx2 & -1.51 & $6.3 \mathrm{E}-05$ & EMSMUSG00000001504 & Iroguois homeobox2 [Source:MGI Symbol;Acc:MGl:1197526] \\
\hline Tbx20 & -1.48 & 1.0E-02 & ENSMUSG00000031965 & T-box 20 [Source:MGI Symbol;Acc:MGl:1888496] \\
\hline Isl2 & -1.47 & 4.0E-08 & ENSMUSG00000032318 & Insulin related protein 2 (islet 2) [Source:MGI Symbol;Acc:MGl:109156] \\
\hline Tррр3 & -1.45 & $1.1 \mathrm{E}-35$ & ENSMUSG00000014846 & $\begin{array}{l}\text { Tubulin polymerization-promoting protein family member3 [Source:MGI } \\
\text { Symbol;Acc:MGl:1915221] }\end{array}$ \\
\hline Sncg & -1.43 & $9.8 \mathrm{E}-66$ & ENSMUSG00000023064 & Synuclein, gamma [Source:MGI Symbol;Acc:MGl:1298397] \\
\hline Hydin & -1.40 & $3.4 \mathrm{E}-02$ & ENSMUSG00000059854 & $\begin{array}{l}\text { HYDIN, axonemal central pair apparatus protein [Source:MGI } \\
\text { Symbol;Acc:MGI:2389007] }\end{array}$ \\
\hline
\end{tabular}

Genes were selected by adjusted $p$-value $<0.05, \mid \log _{2}$ FoldChangel $>0$ and FPKM $>0$. 
TABLE 2 | Transcription factors regulated by ONC injury.

\begin{tabular}{|c|c|c|c|c|}
\hline Gene name & $\begin{array}{l}\log _{2} \\
\text { FoldChange }\end{array}$ & $p$-value & Gene id & Gene description \\
\hline Atf3 & 2.75 & $1.02 \mathrm{E}-67$ & ENSMUSG00000026628 & Activating transcription factor3 [Source:MGI Symbol;Acc:MGl:109384] \\
\hline Sox11 & 2.68 & $2.05 E-48$ & ENSMUSG00000063632 & SRY (sex determining region Y]-box 11 [Source:MGI Symbol;Acc:MGl:98359] \\
\hline Arid5a & 1.45 & 8.81E-29 & ENSMUSG00000037447 & AT rich interactive domain 5A (MRFI-like) [Source:MGI Symbol;Acc:MGI:2443039] \\
\hline Egr3 & 1.29 & $1.58 \mathrm{E}-02$ & ENSMUSG00000033730 & Early growth response3 [Source:MGI Symbol;Acc:MGl:1306780] \\
\hline Atf5 & 1.19 & $1.99 \mathrm{E}-28$ & ENSMUSG00000038539 & Activating transcription factor 5 [Source:MGI Symbol;Acc:MGl:2141857] \\
\hline Creb5 & 1.17 & 2.48E-04 & ENSMUSG00000053007 & cAMP responsive element binding protein 5 [Source:MGI Symbol;Acc:MGl:2443973] \\
\hline Cebpb & 1.13 & $1.79 \mathrm{E}-03$ & ENSMUSG00000056501 & CCAAT/enhancer binding protien (C/EBP), beta [Source [731 Symbo;Acc:MGl:88373] \\
\hline Ddit3 & 0.87 & 9.19E-28 & ENSMUSG00000025408 & DNA-damage inducible transcript 3 [Source:MGI Symbol;Acc:MGl:109247] \\
\hline Fosl2 & 0.65 & 7.05E-05 & ENSMUSG00000029135 & Fos-like antigen 2 [Source:MGI Symbol;Acc:MGl:102858] \\
\hline Jun & 0.46 & 3.36E-13 & ENSMUSG00000052684 & Jun proto-oncogene [Source:MGI Symbol;Acc:MGl:96646] \\
\hline Klf6 & 0.46 & 5.76E-03 & ENSMUSG00000000078 & Kruppel-like factor 6 [Source:MGI Symbol;Acc:MGl:1346318] \\
\hline Bcl6 & 0.46 & 3.35E-02 & ENSMUSG00000022508 & B cell leukemia/lymphoma 6 [Source:MGI Symbol;Acc:MGl:107187] \\
\hline Tead3 & 0.38 & $1.20 E-02$ & ENSMUSG00000002249 & TEA domain family member 3 [Source:MGI Symbol;Acc:MGl:109241] \\
\hline Bhlhe40 & 0.31 & 1.19E-02 & ENSMUSG00000030103 & Basic helix-loop-helix family, member e40 [Source:MGI Symbol;Acc:MGl:1097714] \\
\hline Atf4 & 0.19 & 4.73E-02 & ENSMUSG00000042406 & Activating transcription factor 4 [Source:MGI Symbol;Acc:MGI:88096] \\
\hline Tsc22d1 & -0.19 & $1.32 \mathrm{E}-02$ & ENSMUSG00000022010 & TSC22 domain family, member I [Source:MGI Symbol;Acc:MGL:109127] \\
\hline Six3 & -0.24 & 1.18E-02 & ENSMUSG00000038805 & Sine oculis-related homeobox 3 [Source:MGI Symbol;Acc:MGl:102764] \\
\hline Sebox & -0.25 & 4.97E-02 & ENSMUSG00000001103 & SEBOX homeobox [Source:MGI Symbol;Acc:MGl:108012] \\
\hline Myt1I & -0.26 & 3.18E-02 & ENSMUSG00000061911 & Myelin transcription factor 1ike [Source:MGI:Symbol;Acc:MGl:1100511] \\
\hline Ebf1 & -0.38 & 2.29E-02 & ENSMUSG00000057098 & Early B cell factor 1 [Source:MGI Symbol;Acc:MGl:95275] \\
\hline Scrt1 & -0.41 & $1.50 \mathrm{E}-07$ & ENSMUSG00000048385 & Scratch family zinc finger 1 [SourceMGI Symbol:Acc:MGL:2176606] \\
\hline $\operatorname{lrx} 6$ & -0.56 & 3.33E-03 & ENSMUSG00000031738 & Iroguois homeobox 6 [Source:MGI Symbol;Acc:MGl:1927642] \\
\hline Eomes & -0.67 & $1.58 \mathrm{E}-02$ & ENSMUSG00000032446 & Eomesodermin [Source:MGI Symbol;Acc:MGl:1201683] \\
\hline Zicl & -0.70 & 2.12E-02 & ENSMUSG00000032368 & Zinc finger protein of the cerebellum 1 [Source:MGI Symbol;Acc:MGl:106683] \\
\hline Pou6f2 & -0.84 & 3.05E-04 & ENSMUSG00000009734 & POU domain, class 6, transcriptionfactor 2 [Source:MGI Symbol;Acc:MGl:2443631] \\
\hline Pou4f1 & -0.92 & $2.79 \mathrm{E}-16$ & ENSMUSG00000048349 & POU domain, class4, transcription factor 1 [Source:MGI Symbol;Acc:MGl:102525] \\
\hline Isl2 & -1.47 & 4.00E-08 & ENSMUSG00000032318 & Insulin related protein 2 (islet 2)[Source:MGI Symbol;Acc:MGl:109156] \\
\hline Tbx20 & -1.48 & $1.02 \mathrm{E}-02$ & ENSMUSG00000031965 & T-box 20 [Source:MGI Symbol;Acc:MGl:1888496] \\
\hline Irx2 & -1.51 & $6.26 \mathrm{E}-05$ & ENSMUSG00000001504 & Iroquois homeobox 2[Source:MGI Symbol;Acc:MGl:1197526] \\
\hline Pou4f2 & -1.88 & $1.561-20$ & ENSMUSG00000031688 & POU domain, class4, transcription factor 2 [Source:MGI Symbol;Acc:MGl:102524] \\
\hline Irx4 & -2.36 & $1.02 \mathrm{E}-14$ & ENSMUSG00000021604 & Iroguois homeobox 4 [Source:MGI Symbol;Acc:MGl:1355275] \\
\hline
\end{tabular}

Transcription factors were selected by adjusted p-value $<0.05, \mid \log _{2}$ FoldChange| $>0$ and FPKM $>0$.

also test the DEGs expression reversed by acupuncture treatment. Results showed that expression of Penk (Preproenkephalin) and Mt3 (Metallothionein-3) were significantly decreased after ONC injury, Penk was reversed by BL1 (ONC-J) treatment and Mt3 was reversed by GB20 (ONC-F) treatment, while sham treatment GV16 (ONC-S) had no effect on expression reversal of both candidate DEGs (Figures 5E,F). Expression of Klf6 (Kruppellike factor 6) and Nav1 (neuron navigator 1) were significantly increased after ONC injury and only GB20 treatment reversed and decreased the expression of candidate genes, while BL1 treatment had no effect on expression change and GV16 sham treatment further increased the expression of candidate genes (Figures 5G,H). All these qRT-PCR results were consistent with RNA-seq analysis and demonstrated that acupuncture treatment regulated retinal transcriptome after ONC injury.

\section{Acupuncture Treatment Promote RGC Survival}

Finally, to test how acupuncture treatment affected actual survival of RCGs after ONC, we collected and immunostained the retinas after ONC alone and ONC with acupuncture treatment at acupoints BL1, GB20, and sham control acupoint GV16. Retinas were co-stained with RGC specific marker-RBPMS and neuron specific marker-Tuj1. Results showed that GB20 acupoint treatment slightly increased RGC survival after optic nerve injury. 27\% of RCGs survived in GB20 acupuncture condition, while only $18 \%$ survived under ONC alone. BL1 and GV16 had no significant effect on RGC survival, which was 18 and 22\%, respectively (Figure 6). Thus, ONC with acupoint GB20 treatment resulted in 9\% more RCGs surviving compared to ONC alone.

\section{DISCUSSION}

In this study, we performed RNA-seq analysis of retinal transcriptome 2 days after ONC.

Axotomy injury induced both upregulation and downregulation of more than eight hundred genes, including many pro-apoptotic molecules and ER stress pathway molecules. Our previous studies showed that both traumatic and chronic 
A

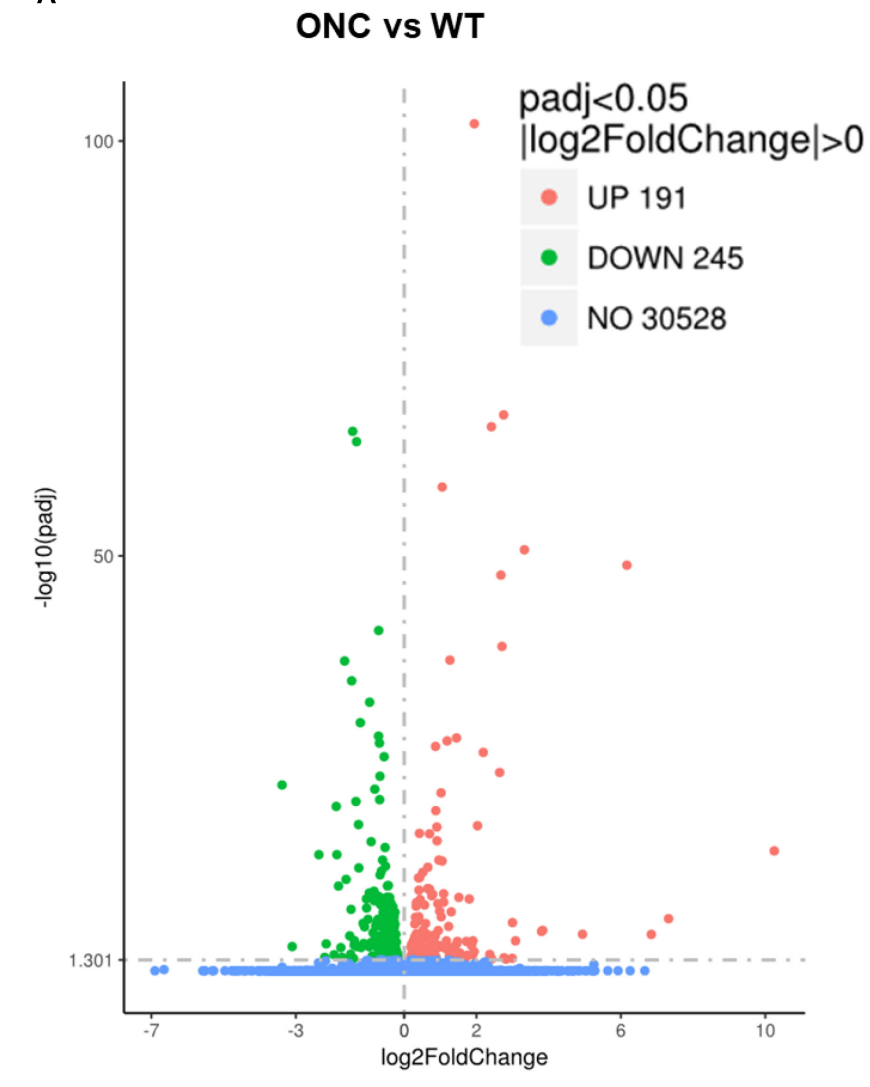

B

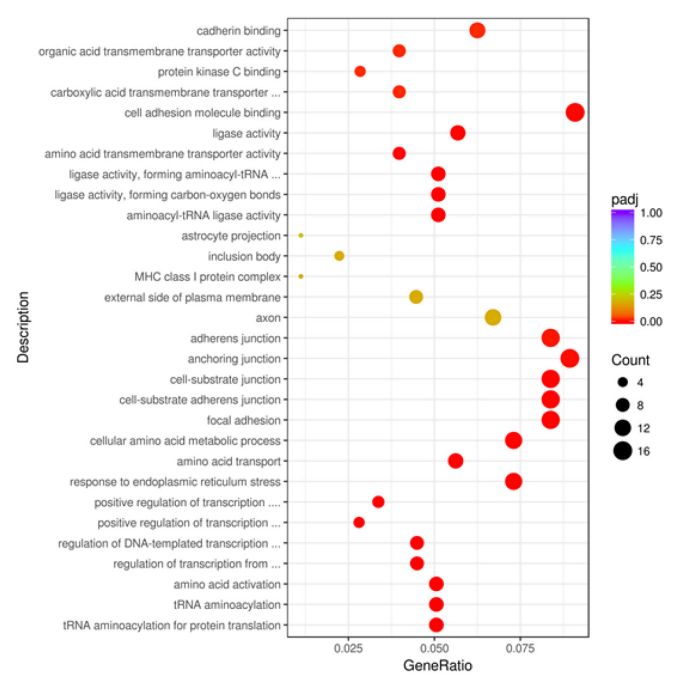

c

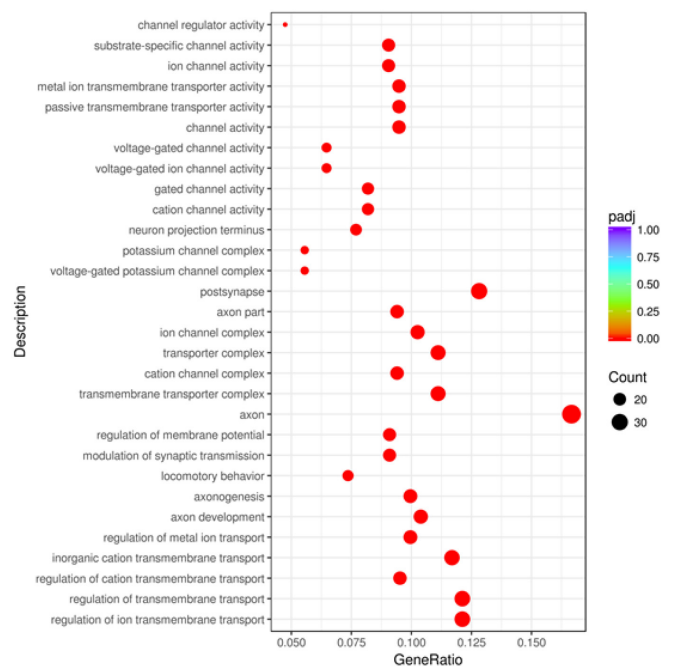

FIGURE 3 | Differentially expressed genes induced by ONC injury. (A) The volcano map shows the overall differentially expressed genes after ONC injury, compared with WT control. (B) Enriched gene ontology analysis of up-regulated DEGs. (C) Enriched gene ontology analysis of down-regulated DEGs. padj: adjusted $p$ value.

neuron injury resulted in activation of PERK-eIF2 $\alpha$-ATF4CHOP pathway or downregulating activity of PI3K-AKT-mTOR pathways, while manipulated these pathways and reversed the injury induced changes resulted in neuroprotection and increased RGC survival (Park et al., 2008; Miao et al., 2016; Yang et al., 2016; Huang et al., 2017, 2019). DLK-JNK-JUN pathway is another important pathway activated by axon injury and knockdown this pathway also resulted in neuroprotection (Kim et al., 2016; Syc-Mazurek et al., 2017b).

In Traditional Chinese Medicine, acupuncture has been broadly used for treatment of ocular diseases. GB20 and BL1 are the most common acupoints for ocular disease treatment. However, BL1 acupoint is too close to the eyeball and it's difficult to handle during clinical practice, and it is the same in mouse acupuncture treatment (Figure 1); GB20 acupoint has many muscles surrounded and it is easy to perform needling at this acupoint. RNA-seq analysis revealed that acupuncture treatment at GB20 reversed the expression of 41 genes and only 6 genes were regulated toward the same direction induced by ONC injury (Figures 4A,D). In BL1 acupoint treatment, 43 genes were reversed, and 13 genes were regulated toward the same direction induced by ONC injury (Figures 4B,E). Although sham treatment at GV16 also reversed 46 genes, 94 genes were regulated toward the same direction induced by ONC injury (Figures 4C,F). As in qRT-PCR results, sham treatment further increased Klf6 and Nav1 expression which were already increased by ONC injury (Figures 5G,H). Therefore, only GB20 treatment had the best overall reversal effect on DEGs expression, and GB20's sham control GV16 treatment had no overall reversal effect at all, in contrast, sham treatment further enhanced the expression states induced by ONC injury. What is more, the promotion of RGC survival by GB20 treatment confirmed the neuroprotection effect of DEG reversal (Figure 6B).

Among these reversal DEG candidates, Mt3 plays an important role in ocular neovascularization and its deficiency will exacerbate retinal degeneration (Tsuruma et al., 2012; 


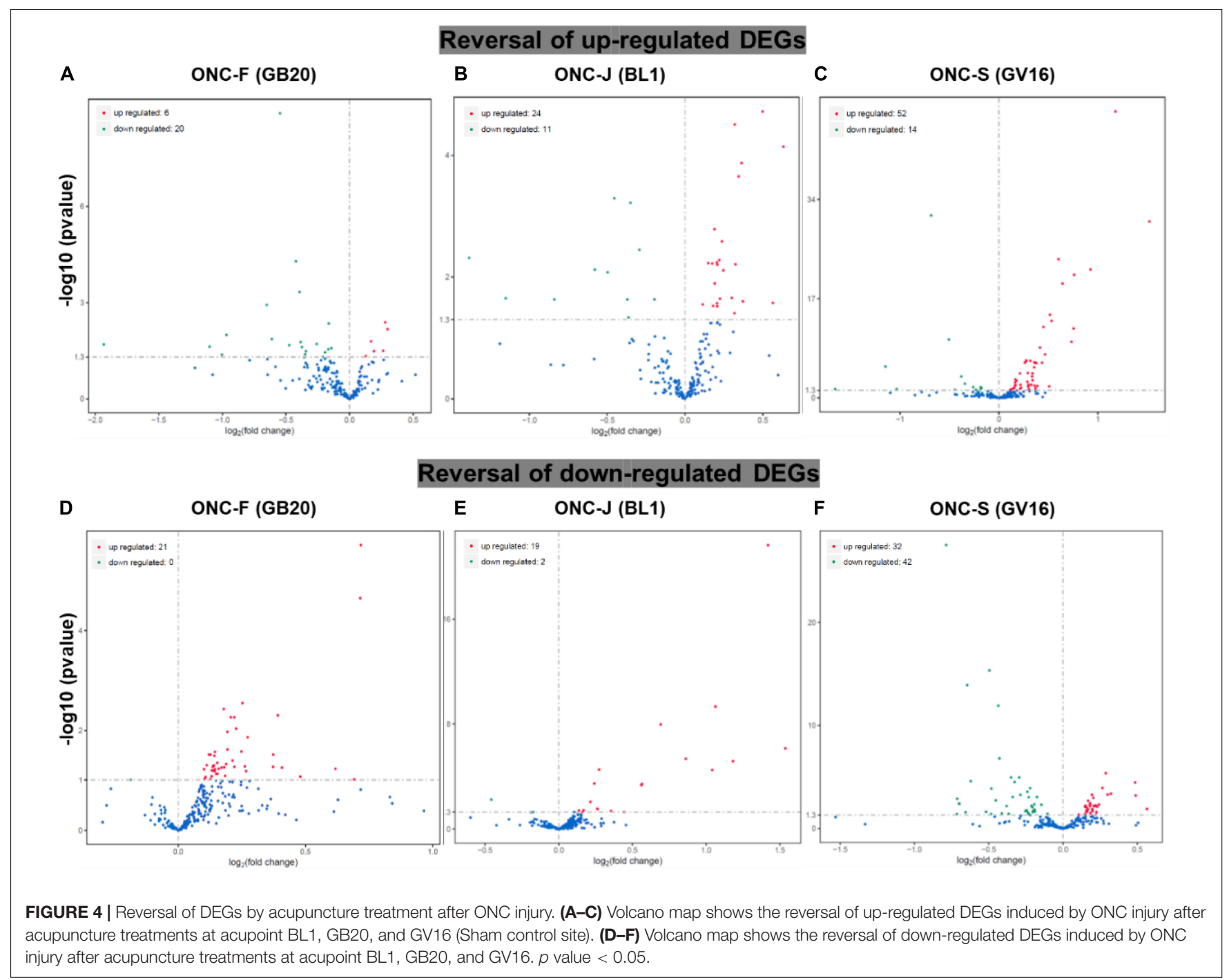

Choi et al., 2013); Fbn1 (fibrillin 1) expression is required for eye development and its mutation is associated with macular degeneration (Hubmacher et al., 2014; Ratnapriya et al., 2014), suggesting that acupuncture treatment may affect vascularization processes in the retina, and promote neuroprotective outcomes after ONC injury. Intriguingly, the site of acupoint GB20 is at the back of the neck, far from the site of eyes, while needling and stimulation at this point surprisingly has effect on regulation of retina gene expression, which explains why many clinical practices choose GB20 site to cure ocular diseases. At last, it is possible that acupuncture treatments relay electrical signal via dorsal root ganglion (DRG) and spinal cord, finally regulate ocular blood flow since many groups reported that acupuncture treatment improved eye blood flow in openangle glaucoma patients (Leszczynska et al., 2018; Vanzini and Gallamini, 2018). It is reported that acupuncture treatment also regulated expression of nerve growth factor and brain-derived neurotrophic factor in retina (Pagani et al., 2006) which may regulate retinal gene expression and provide neuroprotection.
Since GB20 treatment slightly increase RGC survival, next we will investigate the detail mechanism under GB20's involvement in RGC protection.

\section{MATERIALS AND METHODS}

\section{Animals}

We perform experiments in 6 weeks old C57BL/6 mice. All animal procedures were performed in accordance with the National Institute of Health guidelines. The protocol was approved by the Animal Care and Use Committee of Peking University.

\section{Optic Nerve Crush}

Mice were anesthetized by xylazine and ketamine based on their body weight $(0.01 \mathrm{mg}$ xylazine/g $+0.08 \mathrm{mg}$ ketamine/g). Optic nerves of both sides were sequentially exposed intraorbitally and crushed with a jeweler's forceps (Dumont \#5; Fine Science Tools) for $2 \mathrm{~s}$ approximately $0.5 \mathrm{~mm}$ behind the eyeball. Care was taken 


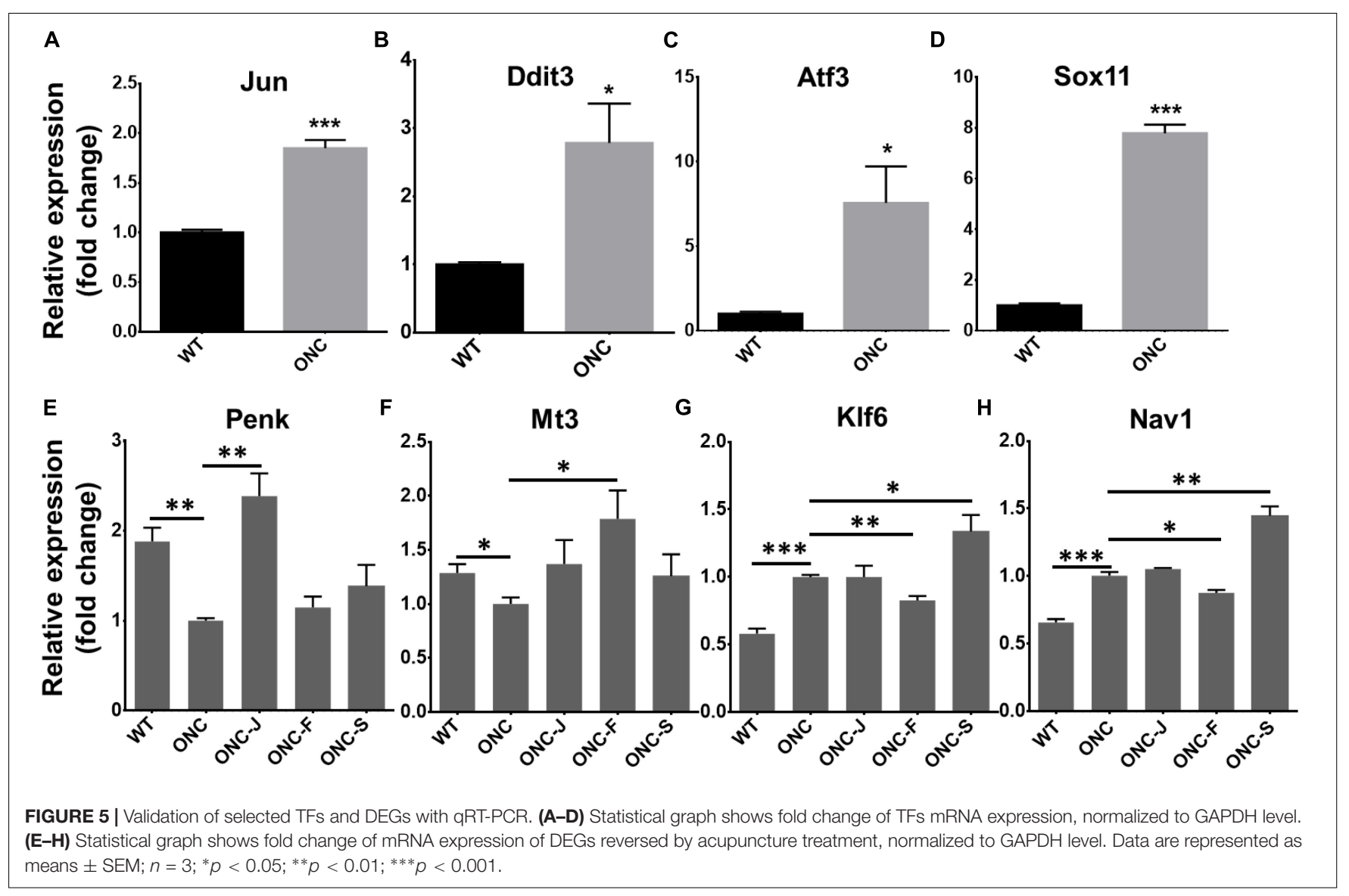

not to damage the underlying ophthalmic artery. Erythromycin Eye Ointment was applied to protect the cornea after surgery.

\section{Acupuncture Treatment}

After optic nerves crush injury, mice were anesthetized by xylazine and ketamine based on their body weight $(0.01 \mathrm{mg}$ xylazine $/ g+0.08 \mathrm{mg}$ ketamine/g) before acupuncture treatment at acupoint GB20 or BL1 at both sides, respectively. The depth of the acupuncture needling is around $2 \mathrm{~mm}$. The duration of acupuncture treatment was $20 \mathrm{~min}$. We then gave another two acupuncture treatments every $24 \mathrm{~h}$.

\section{RNA Preparation}

Mice were randomly divided into four groups (3 mice/group). Experiments were repeated for 3 times. Briefly, in each replicate, mice were sacrificed 2 days after ONC injury, and retinas were dissected out in HBSS buffer (Cellgro) immediately. Retinas were then homogenized with TRIzol Reagent (Thermo Fisher Scientific), and total RNA was extracted from the homogenized mixture according to the reagent instructions. RNA purity was checked using the NanoPhotometer ${ }^{\circledR}$ spectrophotometer (IMPLEN). RNA concentration was measured using Qubit ${ }^{\circledR}$ RNA Assay Kit in Qubit ${ }^{\circledR}$ 2.0 Flurometer (Life Technologies). RNA integrity was assessed using the RNA Nano 6000 Assay Kit of the Bioanalyzer 2100 system (Agilent Technologies).

\section{Library Preparation and Sequencing}

About $3000 \mathrm{ng}$ total RNA generated from each group was used for RNA-seq, which was done at Novogene, Inc. Briefly, RNA samples from three biological replicates went through mRNA purification with poly-T oligo-attached magnetic beads. Sequencing libraries were generated using NEBNext ${ }^{\circledR}$ UltraTM RNA Library Prep Kit for Illumina ${ }^{\circledR}$ (NEB) following manufacturer's recommendations and index codes were added to attribute sequences to each sample. The library fragments were purified with AMPure XP system (Beckman Coulter) for cDNA fragments of preferentially 250 300 bp in length. Library quality was assessed on the Agilent Bioanalyzer 2100 system. The clustering of the index-coded samples was performed on a cBot Cluster Generation System using TruSeq PE Cluster Kit v3-cBot-HS (Illumia) according to the manufacturer's instructions. After cluster generation, the library preparations were sequenced on an Illumina Hiseq platform and $125 \mathrm{bp} / 150 \mathrm{bp}$ paired-end reads were generated.

\section{Gene Expression Analysis}

The RNA-seq reads were aligned to the reference genome using Hisat2 v2.0.5. FeatureCounts v1.5.0-p3 was used to count the reads numbers mapped to each gene. And then FPKM of each gene was calculated based on the length of the gene and reads count mapped to this gene, which normalizes 

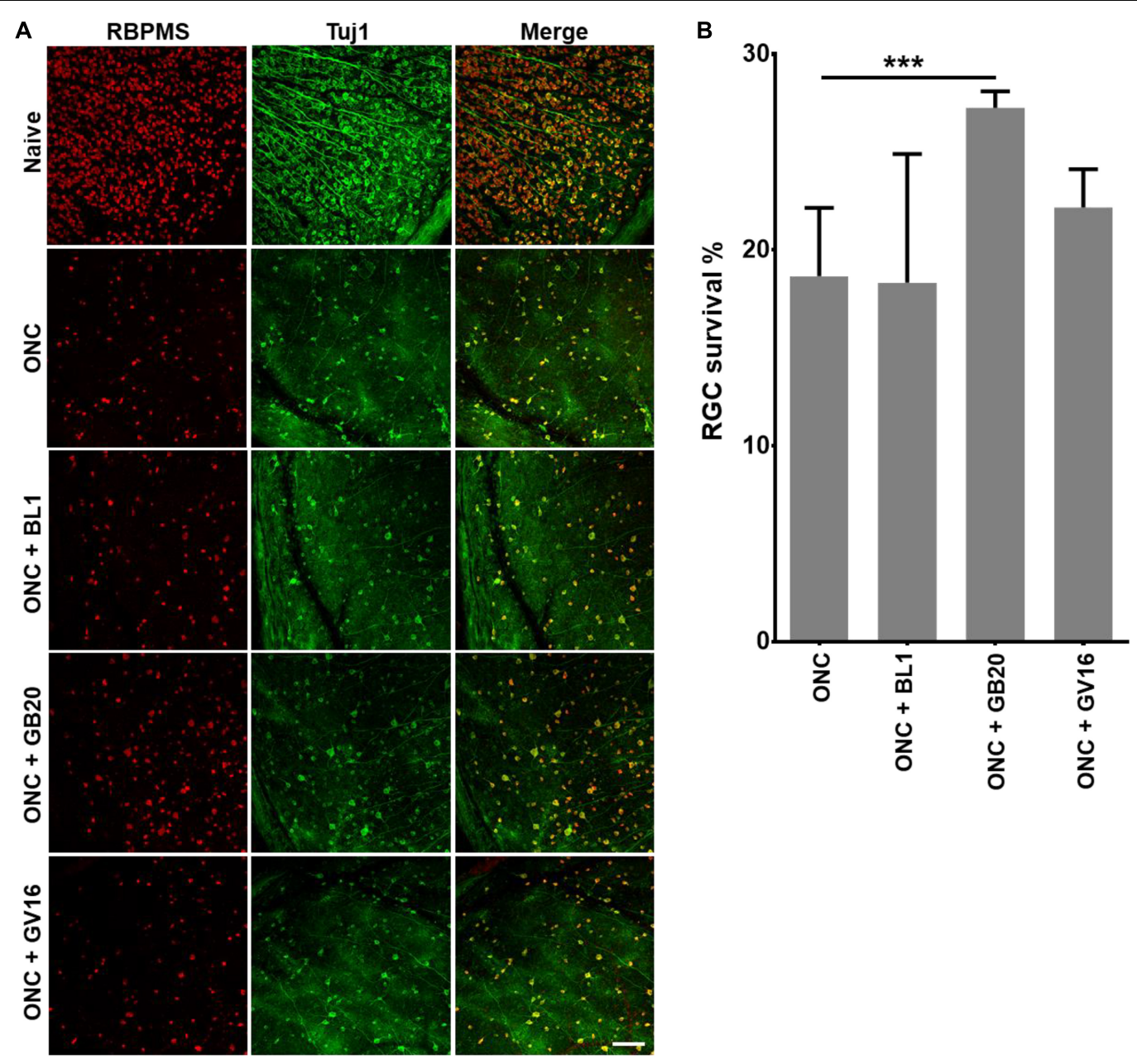

FIGURE 6 | Promotion of RGC survival by acupuncture treatment. (A) Confocal images showing the co-staining of RGC with antibodies of RBMPS (RGC specific marker) and Tuj1 (neuron specific marker). (B) Statistical analysis of survival RGC percentage relative to intact naïve retina. Data are represented as means \pm SEM; $n=5 ;{ }^{* * *} p<0.001$.

gene expression by considering the effect of sequencing depth and gene transcript length at the same time. Differential expression analysis was performed using the DESeq2 R package (1.16.1). The resulting $p$-value were adjusted using the Benjamini and Hochberg's approach for controlling the false discovery rate (less than 0.05). Genes with an adjusted $p$-value $<0.05$ (detected by DESeq2) were considered to be differentially expressed.

\section{Quantitative Real-Time PCR (qRT-PCR)}

Twelve RNA samples (3 samples each group) were examined with qRT-PCR. $1 \mu \mathrm{g}$ total RNA from each sample was reversetranscribed into cDNA using SuperScript III (Invitrogen). All qRT-PCR assays were performed in duplicate. Reactions were carried out with SYBR ${ }^{\circledR}$ Premix Ex Taq ${ }^{\mathrm{TM}}$ kit (TaKaRa) and performed on LightCycler ${ }^{\circledR} 480$ System (Roche). For comparison of relative gene expression, we analyzed qRT-PCR data by $\Delta \mathrm{Ct}$ method and normalized value to endogenous GAPDH control.

\section{Immunostaining of Flat-Mount Retina}

Retinas were dissected out from 4\% PFA fixed eyes and washed extensively in PBS before blocking in staining buffer (10\% normal goat serum and 2\% Triton X-100 in PBS) for $30 \mathrm{~min}$. Mouse neuronal class B-III tubulin (clone Tuj1, 1:200; Biolegend), rabbit RBPMS (GTX118619, 1:400, GeneTex) were diluted in the same staining buffer. Floating retinas were incubated with primary antibodies overnight at $4^{\circ} \mathrm{C}$ and washed three times for 30 min each with PBS. Secondary antibodies (Cy2 and Cy3conjugated) were then applied (1:200; Jackson ImmunoResearch) and incubated for $1 \mathrm{~h}$ at room temperature. Retinas were again washed three times for $30 \mathrm{~min}$ each with PBS before a cover slip was attached with Fluoromount-G (Southernbiotech).

\section{Counting Surviving RGCs}

For RGC counting, whole-mount retinas were immunostained with RBPMS antibody, and 4-6 fields were randomly sampled from peripheral regions of each retina. The percentage of RGC survival was calculated as the ratio 
of surviving RGC numbers in injured eyes compared to contralateral uninjured eyes.

\section{Statistical Analysis}

Data are represented as means \pm SEM. qRT-PCR data was analyzed with Student's $t$-test, and $P$-value $<0.05$ was considered as statistically significant. The raw data and GEO accession number for this study are as follows: GSE131486, link: https://www.ncbi.nlm.nih.gov/geo/query/acc. cgi? acc $=$ GSE131486.

\section{DATA AVAILABILITY STATEMENT}

The datasets generated for this study can be accessed on NCBI website via GEO accession number: GSE131486.

\section{ETHICS STATEMENT}

All animal procedures were performed in accordance with the National Institutes of Health guidelines.

\section{REFERENCES}

Almasieh, M., Wilson, A. M., Morquette, B., Cueva Vargas, J. L., and Di Polo, A. (2012). The molecular basis of retinal ganglion cell death in glaucoma. Prog. Retin. Eye Res. 31, 152-181. doi: 10.1016/j.preteyeres.2011.11.002

Bellon, A., Luchino, J., Haigh, K., Rougon, G., Haigh, J., Chauvet, S., et al. (2010). VEGFR2 (KDR/Flk1) signaling mediates axon growth in response to semaphorin 3E in the developing brain. Neuron 66, 205-219. doi: 10.1016/j. neuron.2010.04.006

Cao, M., Fei, C., Ni, X., Meng-Yao, C., Pengfei, C., Qi, L., et al. (2018). c-Jun $\mathrm{N}$-terminal kinases differentially regulate TNF- and TLRs-mediated necroptosis through their kinase-dependent and -independent activities. Cell Death Dis. 9:1140. doi: 10.1038/s41419-018-1189-2

Choi, J. A., Hwang, J. U., Yoon, Y. H., and Koh, J. Y. (2013). Methallothionein3 contributes to vascular endothelial growth factor induction in a mouse model of choroidal neovascularization. Metallomics 5, 1387-1396. doi: 10.1039/ c3mt00150d

Fagoe, N. D., Attwell, C. L., Kouwenhoven, D., Verhaagen, J., and Mason, M. R. (2015). Overexpression of ATF3 or the combination of ATF3, c-Jun, STAT3 and Smad1 promotes regeneration of the central axon branch of sensory neurons but without synergistic effects. Hum. Mol. Genet. 24, 6788-6800. doi: 10.1093/ hmg/ddv383

Hiramatsu, N., Messah, C., Han, J., LaVail, M. M., Kaufman, R. J., and Lin, J. H. (2014). Translational and posttranslational regulation of XIAP by eIF2alpha and ATF4 promotes ER stress-induced cell death during the unfolded protein response. Mol. Biol. Cell 25, 1411-1420. doi: 10.1091/mbc.E13-11-0664

Holland, S. M., Collura, K. M., Ketschek, A., Noma, K., Ferguson, T. A., Jin, Y., et al. (2016). Palmitoylation controls DLK localization, interactions and activity to ensure effective axonal injury signaling. Proc. Natl. Acad. Sci. U.S.A. 113, 763-768. doi: 10.1073/pnas.1514123113

Hu, Y., Park, K. K., Yang, L., Wei, X., Yang, Q., Cho, K. S., et al. (2012). Differential effects of unfolded protein response pathways on axon injury-induced death of retinal ganglion cells. Neuron 73, 445-452. doi: 10.1016/j.neuron.2011. 11.026

Huang, H., Miao, L., Liang, F., Liu, X., Xu, L., Teng, X., et al. (2017). Neuroprotection by eIF2alpha-CHOP inhibition and XBP-1 activation in EAE/optic neuritiss. Cell Death Dis. 8:e2936. doi: 10.1038/cddis.2017.329

Huang, H., Miao, L., Yang, L., Liang, F., Wang, Q., Zhuang, P., et al. (2019). AKT-dependent and -independent pathways mediate PTEN deletion-induced

\section{AUTHOR CONTRIBUTIONS}

LM and XL designed the experiments. LM, XL, JC, LZha, LL, XY, FW, XG, RZ, YH, QL, HF, LZho, JZ, and AL performed the experiments, and collected and analyzed the data. LM prepared the figures. LM and XL prepared the manuscript.

\section{FUNDING}

This work was supported by the National Natural Science Foundation of China (NSFC) (81803856). Portions of this work were supported by the Beijing Institute of Technology Research Fund Program for Young Scholars and Beijing University of Chinese Medicine Research Fund for Young Scholars.

\section{SUPPLEMENTARY MATERIAL}

The Supplementary Material for this article can be found online at: https://www.frontiersin.org/articles/10.3389/fnint. 2019.00059/full\#supplementary-material

CNS axon regeneration. Cell Death Dis. 10:203. doi: 10.1038/s41419-0181289-Z

Hubmacher, D., Reinhardt, D. P., Plesec, T., Schenke-Layland, K., and Apte, S. S. (2014). Human eye development is characterized by coordinated expression of fibrillin isoforms. Invest. Ophthalmol. Vis. Sci. 55, 7934-7944. doi: 10.1167/iovs. 14- 15453

Ishizawa, J., Kojima, K., Chachad, D., Ruvolo, P., Ruvolo, V., Jacamo, R. O., et al. (2016). ATF4 induction through an atypical integrated stress response to ONC201 triggers p53-independent apoptosis in hematological malignancies. Sci. Signal. 9:ra17. doi: 10.1126/scisignal.aac4380

Jiao, N. J. (2011). [Observation on therapeutic effect of age-related macular degeneration treated with acupuncture]. Zhongguo Zhen Jiu 31, 43-45.

Joo, J. H., Ueda, E., Bortner, C. D., Yang, X. P., Liao, G., and Jetten, A. M. (2015). Farnesol activates the intrinsic pathway of apoptosis and the ATF4-ATF3CHOP cascade of ER stress in human T lymphoblastic leukemia Molt4 cells. Biochem. Pharmacol. 97, 256-268. doi: 10.1016/j.bcp.2015.08.086

Kay, J. C., Miao, L., Shanwei, S., Sharon, J. Y., Chulwon, C., Li-Ya, Q., et al. (2013). Endogenous PI3K/Akt and NMDAR act independently in the regulation of CREB activity in lumbosacral spinal cord in cystitis. Exp. Neurol. 250, 366-375. doi: 10.1016/j.expneurol.2013.10.015

Kim, B. J., Sean, M. S., Yang, L., Robert, J. W., Iok-Hou, P., and Abbot, F. C. (2016). In vitro and in vivo neuroprotective effects of cJun N-terminal kinase inhibitors on retinal ganglion cells. Mol. Neurodegener. 11:30. doi: 10.1186/s13024-0160093-4

Koh, W., Kang, K., Lee, Y. J., Kim, M. R., Shin, J. S., Lee, J., et al. (2018). Impact of acupuncture treatment on the lumbar surgery rate for low back pain in Korea: a nationwide matched retrospective cohort study. PLoS One 13:e0199042. doi: 10.1371/journal.pone.0199042

Law, S. K., and Li, T. (2013). Acupuncture for glaucoma. Cochrane Database Syst. Rev. 5:CD006030. doi: 10.1002/14651858.CD006030.pub3

Leszczynska, A., Ramm, L., Spoerl, E., Pillunat, L. E., and Terai, N. (2018). The short-term effect of acupuncture on different ocular blood flow parameters in patients with primary open-angle glaucoma: a randomized, clinical study. Clin. Ophthalmol. 12, 1285-1291. doi: 10.2147/OPTH.S170396

Li, Y., Struebing, F. L., Wang, J., King, R., and Geisert, E. E. (2018). Different effect of Sox11 in retinal ganglion cells survival and axon regeneration. Front. Genet. 9:633. doi: 10.3389/fgene.2018.00633

Lu, T. Y., Jennifer, M. M., Lukas, J. N., Amy, E. S., Rachel, B., Mary, A. L., et al. (2017). Axon degeneration induces glial responses through 
Draper-TRAF4-JNK signalling. Nat. Commun. 8:14355. doi: 10.1038/ncomms1 4355

MacPherson, H., Vertosick, E. A., Foster, N. E., Lewith, G., Linde, K., Sherman, K. J., et al. (2017). The persistence of the effects of acupuncture after a course of treatment: a meta-analysis of patients with chronic pain. Pain 158, 784-793. doi: 10.1097/j.pain.0000000000000747

Mayer, T. Z., Simard, F. A., Cloutier, A., Vardhan, H., Dubois, C. M., and McDonald, P. P. (2013). The p38-MSK1 signaling cascade influences cytokine production through CREB and C/EBP factors in human neutrophils. J. Immunol. 191, 4299-4307. doi: 10.4049/jimmunol.1301117

Miao, L., Yang, L., Huang, H., Liang, F., Ling, C., and Hu, Y. (2016). mTORC1 is necessary but mTORC2 and GSK3beta are inhibitory for AKT3-induced axon regeneration in the central nervous system. eLife 5:e14908. doi: 10.7554/eLife. 14908

Norsworthy, M. W., Bei, F., Kawaguchi, R., Wang, Q., Tran, N. M., Li, Y., et al. (2017). Sox11 expression promotes regeneration of some retinal ganglion cell types but kills others. Neuron 94, 1112.e4-1120.e4. doi: 10.1016/j.neuron.2017. 05.035

Pagani, L., Manni, L., and Aloe, L. (2006). Effects of electroacupuncture on retinal nerve growth factor and brain-derived neurotrophic factor expression in a rat model of retinitis pigmentosa. Brain Res. 1092, 198-206. doi: 10.1016/j.brainres. 2006.03.074

Park, K. K., Liu, K., Hu, Y., Smith, P. D., Wang, C., Cai, B., et al. (2008). Promoting axon regeneration in the adult CNS by modulation of the PTEN/mTOR pathway. Science 322, 963-966. doi: 10.1126/science.1161566

Qin, Y., Yuan, W., Deng, H., Xiang, Z., Yang, C., Kou, X., et al. (2015). Clinical efficacy observation of acupuncture treatment for nonarteritic anterior ischemic optic neuropathy. Evid. Based Complement. Alternat. Med. 2015:713218. doi: 10.1155/2015/713218

Ratnapriya, R., Zhan, X., Fariss, R. N., Branham, K. E., Zipprer, D., Chakarova, C. F., et al. (2014). Rare and common variants in extracellular matrix gene Fibrillin 2 (FBN2) are associated with macular degeneration. Hum. Mol. Genet. 23, 5827-5837. doi: 10.1093/hmg/ddu276

Sanchez-Migallon, M. C., Valiente-Soriano, F. J., Nadal-Nicolas, F. M., Vidal-Sanz, M., and Agudo-Barriuso, M. (2016). Apoptotic retinal ganglion cell death after optic nerve transection or crush in mice: delayed RGC loss with BDNF or a caspase 3 inhibitor. Invest. Ophthalmol. Vis. Sci. 57, 81-93. doi: 10.1167/iovs. $15-17841$

Shindler, K. S., Ventura, E., Dutt, M., and Rostami, A. (2008). Inflammatory demyelination induces axonal injury and retinal ganglion cell apoptosis in experimental optic neuritis. Exp. Eye Res. 87, 208-213. doi: 10.1016/j.exer.2008. 05.017

Struebing, F. L., Wang, J., Li, Y., King, R., Mistretta, O. C., English, A. W., et al. (2017). Differential expression of Sox11 and Bdnf mRNA isoforms in the injured and regenerating nervous systems. Front. Mol. Neurosci. 10:354. doi: 10.3389/fnmol.2017.00354

Syc-Mazurek, S. B., Fernandes, K. A., and Libby, R. T. (2017a). JUN is important for ocular hypertension-induced retinal ganglion cell degeneration. Cell Death Dis. 8:e2945. doi: $10.1038 /$ cddis.2017.338
Syc-Mazurek, S. B., Fernandes, K. A., Wilson, M. P., Shrager, P., and Libby, R. T. (2017b). Together JUN and DDIT3 (CHOP) control retinal ganglion cell death after axonal injury. Mol. Neurodegener. 12:71. doi: 10.1186/s13024-0170214-8

Tsuruma, K., Shimazaki, H., Ohno, Y., Inoue, Y., Honda, A., Imai, S., et al. (2012). Metallothionein-III deficiency exacerbates light-induced retinal degeneration. Invest. Ophthalmol. Vis. Sci. 53, 7896-7903. doi: 10.1167/iovs.12-10165

Vanzini, M., and Gallamini, M. (2018). Laser acupuncture in open-angle glaucoma treatment a retrospective study of eye blood flow. J. Acupunct. Meridian Stud. doi: 10.1016/j.jams.2018.11.005 [Epub ahead of print].

Walter, F., O’Brien, A., Concannon, C. G., Dussmann, H., and Prehn, J. H. M. (2018). ER stress signaling has an activating transcription factor 6alpha (ATF6)dependent "off-switch". J. Biol. Chem. 293, 18270-18284. doi: 10.1074/jbc. RA118.002121

Welsbie, D. S., Mitchell, K. L., Jaskula-Ranga, V., Sluch, V. M., Yang, Z., Kim, J., et al. (2017). Enhanced functional genomic screening identifies novel mediators of dual leucine zipper kinase-dependent injury signaling in neurons. Neuron 94 , 1142.e6-1154.e6. doi: 10.1016/j.neuron.2017.06.008

Wong, A. W., Osborne, P. B., and Keast, J. R. (2018). Axonal injury induces ATF3 in specific populations of sacral preganglionic neurons in male rats. Front. Neurosci. 12:766. doi: 10.3389/fnins.2018.00766

Woo, C. W., Kutzler, L., Kimball, S. R., and Tabas, I. (2012). Toll-like receptor activation suppresses ER stress factor $\mathrm{CHOP}$ and translation inhibition through activation of eIF2B. Nat. Cell Biol. 14, 192-200. doi: 10.1038/ncb2408

Wu, H. B., Yang, S., Weng, H. Y., Chen, Q., Zhao, X. L., Fu, W. J., et al. (2017). Autophagy-induced KDR/VEGFR-2 activation promotes the formation of vasculogenic mimicry by glioma stem cells. Autophagy 13, 1528-1542. doi: $10.1080 / 15548627.2017 .1336277$

Xu, H., Wang, S., and Guo, M. H. (2012). [ZHANG Ren's experience of treatment on glaucoma with acupuncture]. Zhongguo Zhen Jiu 32, 444-447.

$\mathrm{Xu}$, J., and Peng, Q. (2015). Retinitis pigmentosa treatment with western medicine and traditional chinese medicine therapies. J. Ophthalmol. 2015:421269. doi: 10.1155/2015/421269

Yang, L., Li, S., Miao, L., Huang, H., Liang, F., Teng, X., et al. (2016). Rescue of glaucomatous neurodegeneration by differentially modulating neuronal endoplasmic reticulum stress molecules. J. Neurosci. 36, 5891-5903. doi: 10.1523/JNEUROSCI.3709-15.2016

Conflict of Interest: The authors declare that the research was conducted in the absence of any commercial or financial relationships that could be construed as a potential conflict of interest.

Copyright (c) 2019 Chen, Zhang, Liu, Yang, Wu, Gan, Zhang, He, Lv, Fu, Zhou, Zhang, Liu, Liu and Miao. This is an open-access article distributed under the terms of the Creative Commons Attribution License (CC BY). The use, distribution or reproduction in other forums is permitted, provided the original author(s) and the copyright owner(s) are credited and that the original publication in this journal is cited, in accordance with accepted academic practice. No use, distribution or reproduction is permitted which does not comply with these terms. 